\title{
Exploration of Self-aggregation of Coumarin 7 and Coumarin 30 in Water: Role of $\beta$-Cyclodextrin as a Modulator
}

\author{
Arindam Das, Sinjan Das, Arnab Biswas, and Nitin Chattopadhyay*
}

Department of Chemistry, Jadavpur University, Kolkata - 700 032, India

*Corresponding author: Fax: 91-33-2414-6584

E-mail: nitin.chattopadhyay@yahoo.com

\section{Supporting Information}
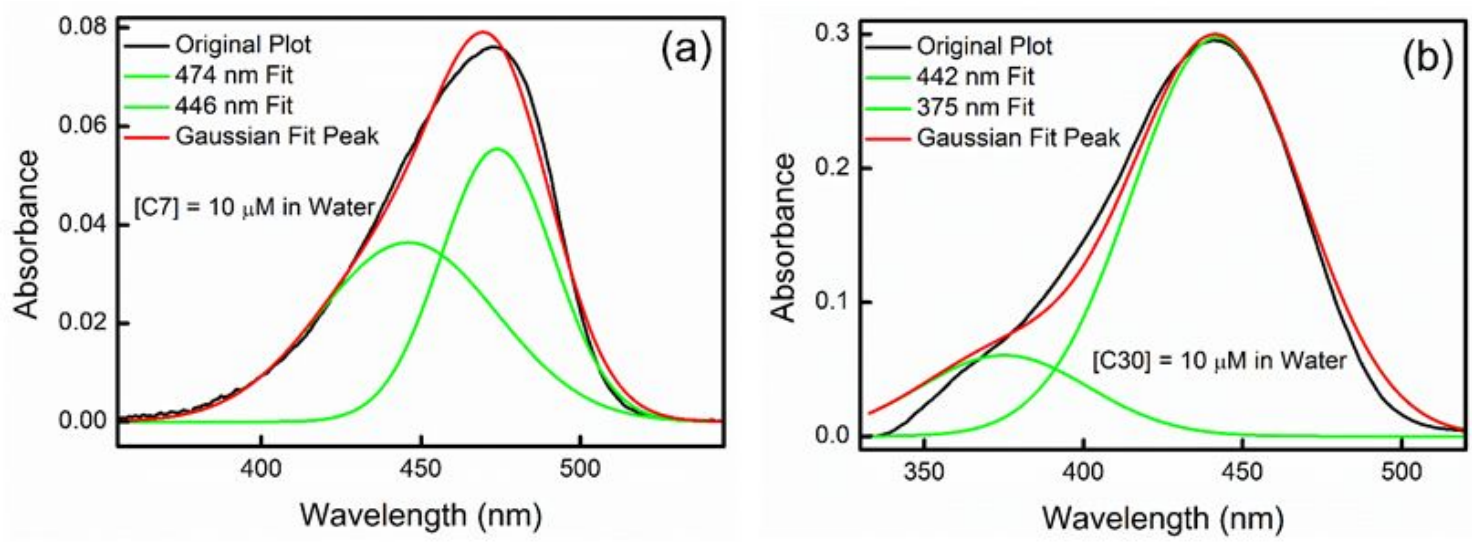

Figure S1. Resolved absorbance spectra of (a) C7 and (b) C30 in water.
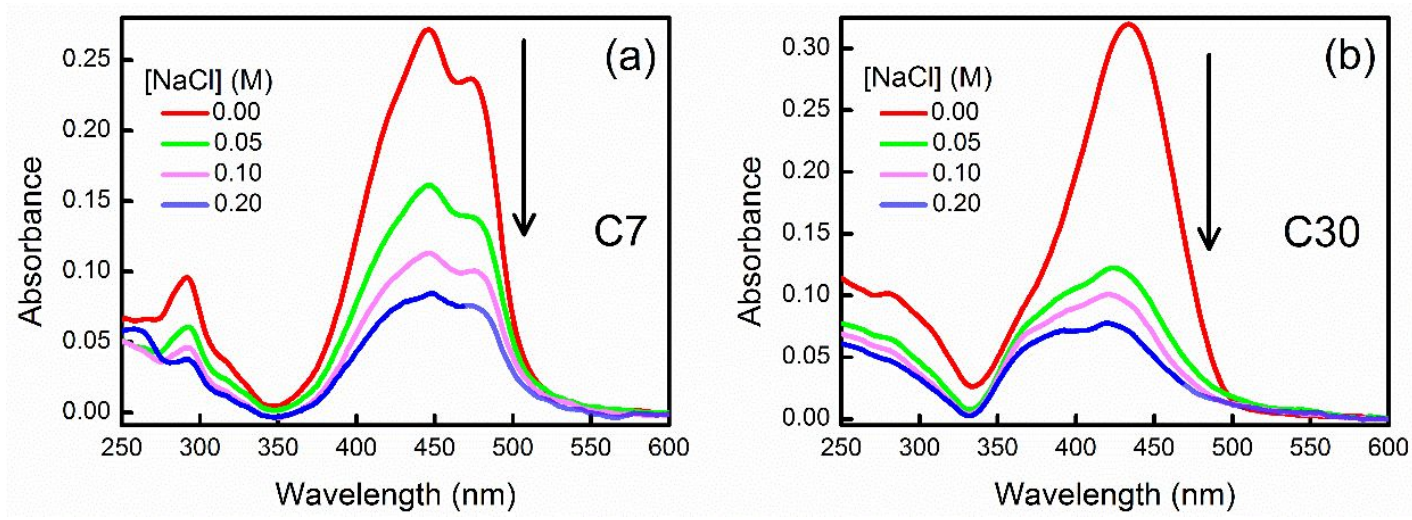

Figure S2. Variation of absorbance spectra of (a) C7 and (b) C30 in water as a function of $\mathrm{NaCl}$ concentration. 

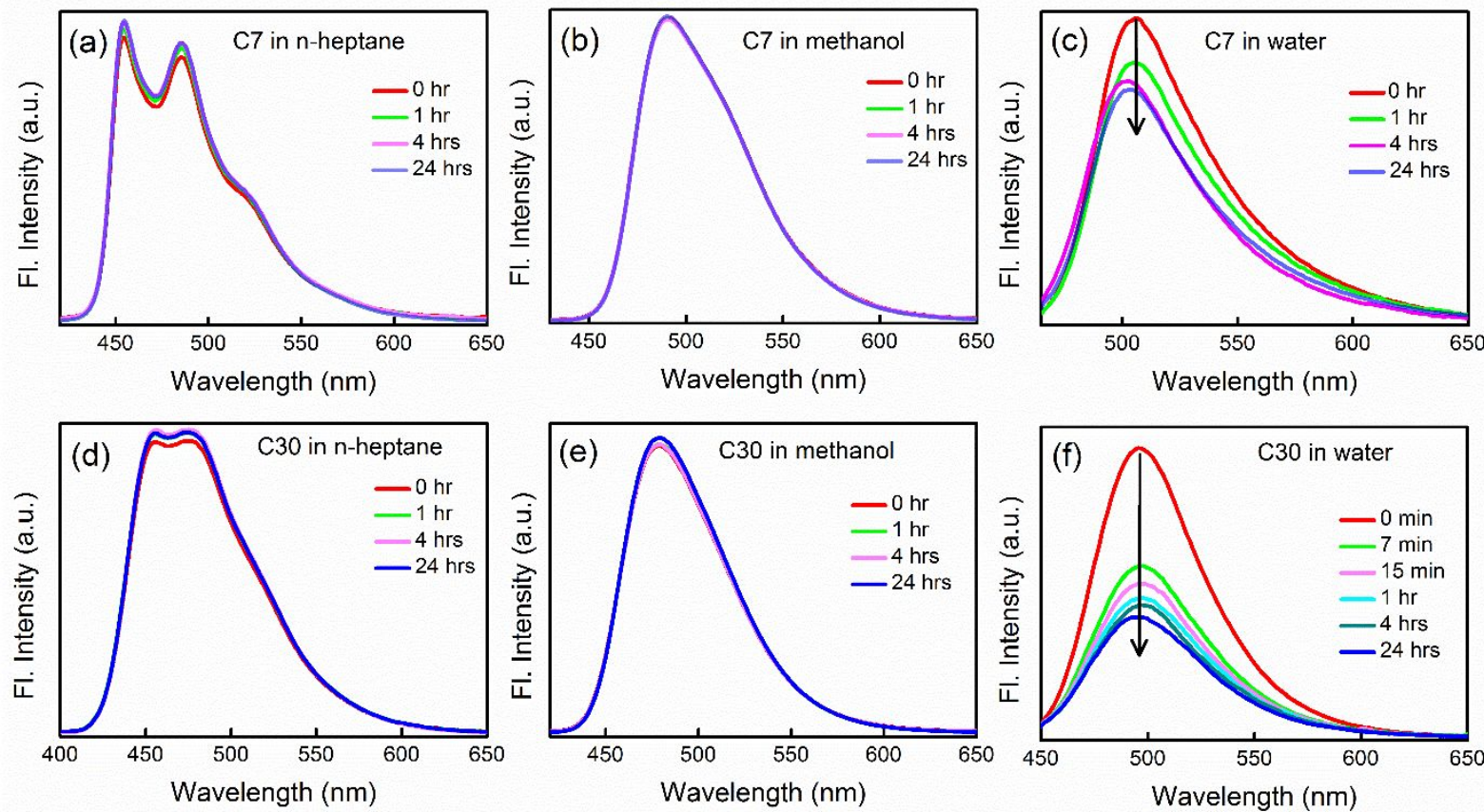

Figure S3. Fluorescence emission spectra of C7 in (a) n-heptane (b) methanol and (c) water at different times. Fluorescence emission spectra of C30 in (d) n-heptane (e) methanol and (f) water at different times.
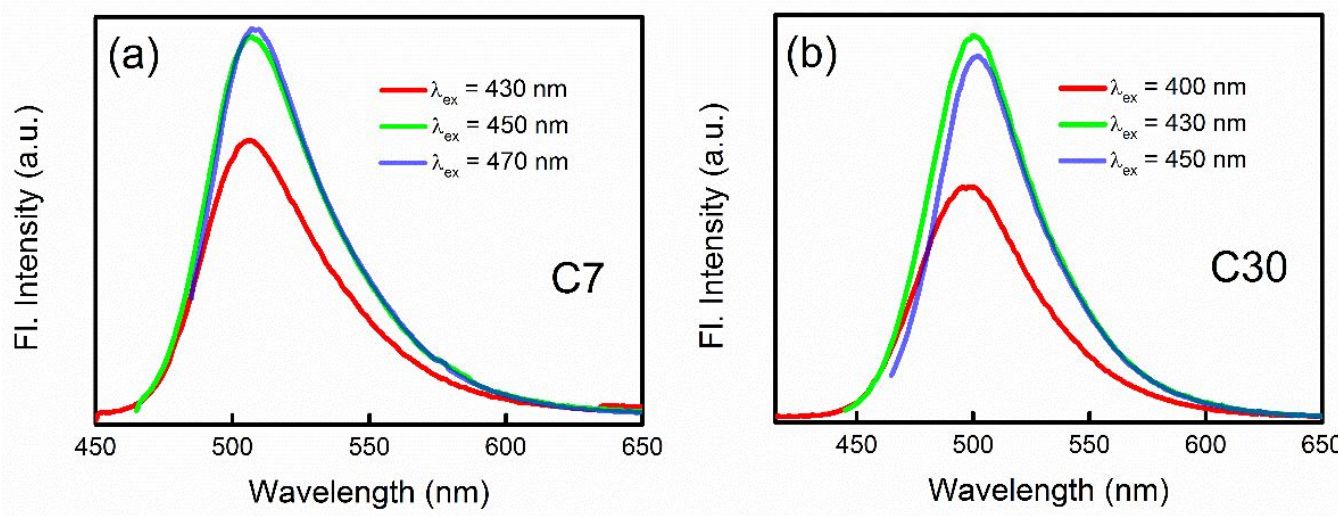

Figure S4. Fluorescence spectra of (a) C7 and (b) C30 in water with variation of excitation wavelength. 

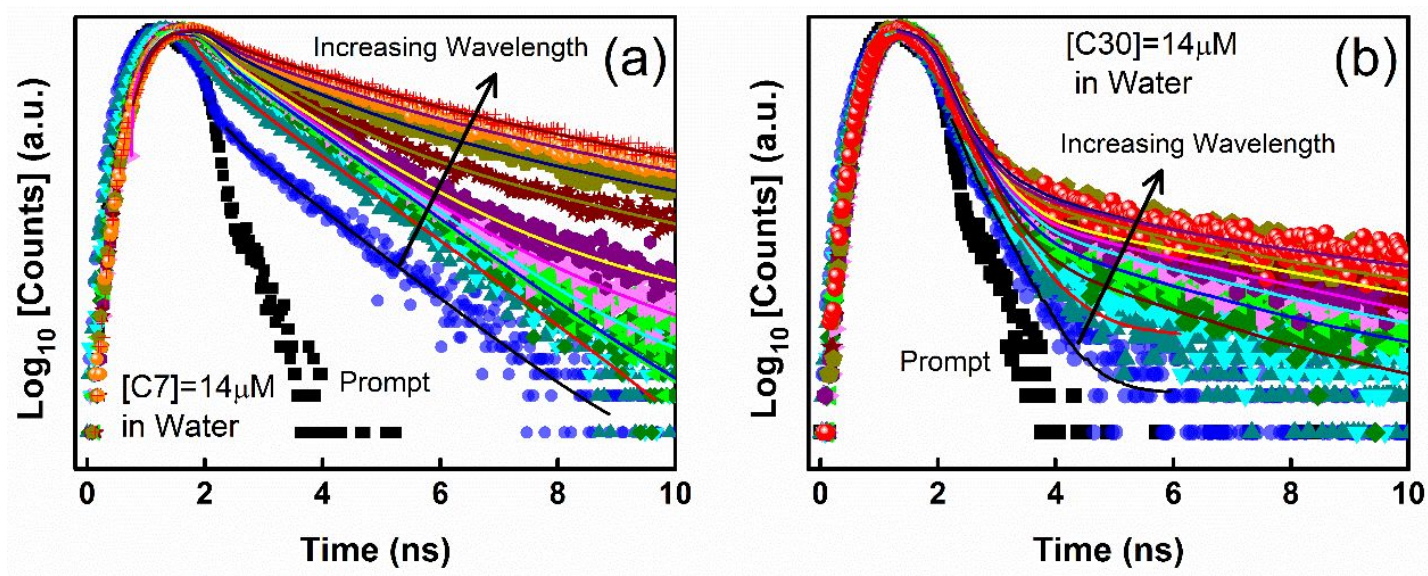

Figure S5. Fluorescence decay traces of (a) C7 and (b) C30 excited at $450 \mathrm{~nm}$ in water at different wavelength of the dye.

Table S1. Hydrodynamic diameter of C7 and C30 solutions in different condition from DLS experiments.

\begin{tabular}{|c|c|}
\hline Samples in aqueous solution & Hydrodynamic Diameter $(\mathbf{n m}) \mathbf{( \pm \mathbf { 0 . 1 } )}$ \\
\hline C7 fresh & 1.33 \\
\hline C7 kept overnight & 2.55 \\
\hline C30 fresh & 1.41 \\
\hline C30 kept overnight & 3.12 \\
\hline$\beta-C D$ only & 1.74 \\
\hline$\beta-C D+C 7$ & 1.89 \\
\hline$\beta-C D+C 30$ & 1.95 \\
\hline
\end{tabular}

Table S2. Zero corrected electronic energies of C7, C30 monomers and dimers calculated by DFT using B3LYP and 6-31 G basis set.

\begin{tabular}{|c|c|c|}
\hline Species & Electronic energy (Hartree) & $\begin{array}{c}\text { Difference in energy between } \\
\text { dimer and a pair of respective } \\
\text { monomers (kJ mol-1) }\end{array}$ \\
\hline C7 monomer & -1088.0392 & \\
\hline C7 dimer & -2176.0805 & 5.51 \\
\hline C30 monomer & -1127.3216 & \\
\hline C30 dimer & -2254.6459 & 7.09 \\
\hline
\end{tabular}

